\title{
Mapping the observable sky for a Remote Occulter working with ground-based telescopes
}

\author{
Eliad Peretz $\odot$, a,* John C. Mather $\odot,{ }^{\text {a }}$ Lucas Pabarcius $\odot,{ }^{\text {a }}$ Sara Seager $\odot,{ }^{b}$ \\ Stuart Shaklan $\odot,{ }^{c}$ Sergi Hildebrandt $\odot,{ }^{c}$ Phil Willems, ${ }^{c}$ and Kevin Hall ${ }^{d}$ \\ ${ }^{a}$ NASA Goddard Space Flight Center, Greenbelt, Maryland, United States \\ ${ }^{b}$ Massachusetts Institute of Technology, Department of Earth, Atmospheric, and \\ Planetary Sciences, Cambridge, Massachusetts, United States \\ ${ }^{c} J e t$ Propulsion Laboratory, California Institute of Technology, Pasadena, \\ California, United States \\ ${ }^{\mathrm{d} U n i v e r s i t y ~ o f ~ M a r y l a n d, ~ D e p a r t m e n t ~ o f ~ A s t r o n o m y, ~ C o l l e g e ~ P a r k, ~ M a r y l a n d, ~ U n i t e d ~ S t a t e s ~}$
}

\begin{abstract}
We present the optical requirement-driven observational constraints of the Remote Occulter, an orbiting starshade designed to work with ground-based telescopes to produce visible-band images and spectra of temperate planets around Sun-like stars. We then utilize these constraints to develop and present numerical simulations of time-dependent observable sky regions along with each region's nightly available exposure duration and show that nearly the entire sky could be observed for up to $8 \mathrm{~h}$ a night. We further examine how changes introduced to our established constraints will impact such observational windows and discuss their implications, setting the ground for upcoming studies aiming to further investigate the Remote Occulter mission capabilities and architecture. (C) 2021 Society of Photo-Optical Instrumentation Engineers (SPIE) [DOI: 10.1117/1.JATIS.7.2.021212]
\end{abstract}

Keywords: starshade; extremely large telescopes; night sky; numerical simulations.

Paper 20114SS received Aug. 1, 2020; accepted for publication Jan. 4, 2021; published online Jan. 25, 2021.

\section{Introduction}

Measuring the reflected light spectra of temperate planets around Sun-like stars has been identified by the exoplanet science community as a high-priority objective. ${ }^{1,2}$ This is a major technological challenge: temperate exoplanets typically reside close to their parent star at $\sim 100$ mas and are extremely faint at 30th Mag, typically $\sim 25$ magnitudes fainter than their parent star. ${ }^{3}$ Parsing temperate exoplanetary signal from its parent star is further complicated by a variety of natural variables including exozodiacal dust ${ }^{4}$ and its planetary phase.

Although some 48 exoplanets have been directly imaged to date, ${ }^{5}$ including some around Sun-like stars, ${ }^{6,7}$ none are rocky worlds; the lowest mass planets yet imaged are $\sim 2 M_{\text {jup. }}{ }^{8}$ Proposed next generation space telescopes including $\mathrm{HabEx}^{9}$ and $\mathrm{LUVOIR}^{10}$ are one promising path toward expanding this catalog, by way of coupling with coronagraphs ${ }^{11,12}$ and/or starshades. ${ }^{13,14}$ However, space telescopes face many cost prohibitions and engineering challenges in exceeding their current sizes, including stability constraints ${ }^{15}$ and launch vehicle fairing diameters. Future extremely large ground-based telescopes with diameters exceeding $20 \mathrm{~m}$, including the Extremely Large Telescope (ELT) (39 m), Thirty Meter Telescope (TMT) (30 m), and Giant Magellan Telescope (GMT) $(24.5 \mathrm{~m})$, will be the largest telescopes in the coming decades, with the first lights currently planned to occur between 2025 and 2030. ${ }^{16-18}$ However, due to engineering, programmatic, and fundamental atmospheric physical constraints, AO and coronagraphassisted ground systems are likely limited to $10^{-8}$ contrast for the foreseeable future. ${ }^{19,20}$

Another approach, marrying an extremely large ground telescope with an orbiting starshade may further enhance exoplanet imaging capabilities. Ground-coupled starshade systems like the Remote Occulter provide smaller inner working angles, better angular resolution, larger observable ranges, are less sensitive to exozodiacal dust, starshade perturbations ${ }^{21,22}$ and are expected to

*Address all correspondence to Eliad Peretz, eliad.peretz@nasa.gov 
deliver contrast ratios exceeding $10^{-10} .{ }^{23}$ Where previous research on the Remote Occulter mission concept has resolved its imaging and spectral capabilities, ongoing efforts are focused on understanding and optimizing its challenging operational scenario, including spacecraft architecture, orbital configurations, and mission schedule, which will require well-defined models of target observability. ${ }^{24,25}$

In this paper, we derive the observational geometry constraints produced by an earth-orbiting starshade, including the starshade plane-Sun angle (Sec. 2.1), the starshade plane-target star angle (Sec. 2.2), observatory zenith-Sun angle (Sec. 2.3), and observatory zenith-target star angle (Sec. 2.4). We then incorporate all requirements alongside time-dependent Sun-Earth ephemerides to produce a sky map that reveals which regions of the sky may be observed with the Remote Occulter at a given date and time, as well as each celestial coordinate's cumulative nightly exposure availability (Sec. 3). Finally, we discuss the operational implications of the mission (Sec. 4) and provide a road map for future work advancing this starshade mission concept (Sec. 5).

\section{Observational Requirements}

To acquire both images and spectra of temperate planets around Sun-like stars, we must mitigate conditions and effects that could interfere with observation. We would ideally like to observe for long periods anywhere in the night sky through most of the year to reduce target star scheduling complexity and provide flexibility in mission planning. However, an array of optical requirement-driven constraints limits the range of favorable operating conditions. For mission planning purposes, it is useful to describe these constraints in geometric terms that can directly translate to the ground telescope's available sky domain and the orbiting starshade's corresponding configuration and orbit. In this section, we establish these geometric requirements, enabling the numerical calculation of a well-defined observable sky region.

\subsection{Starshade Plane-Sun Angle}

We would like to minimize sunlight illumination of the Earth-facing starshade surface, as the reflected light could interfere with observations. We define the starshade plane-Sun angle $\theta_{S-\odot}$ in Fig. 1 and assess the apparent magnitude of the starshade surface at various relative starshade-Sun angles in Fig. 2. We find that direct illumination can produce a Mag. 8 to 14 starshade, restricting both image and spectral capabilities, additional information is provided in Appendix B. Starshade brightness can be further reduced using a low-reflectance carbon nanotube coating under reduced starshade-Sun angles $\theta_{S-\odot}$, producing between 15 and $18 \mathrm{Mag}$. This may impact the detection of low-separation exoplanets, pending future performance simulations.

To avoid these effects, we constrain the Sun to be behind the earth-facing starshade surface by at least one degree, corresponding to a starshade-Sun tilt requirement $\theta_{S-\odot}$ of $89 \mathrm{deg}$. Following this constraint, we expect the primary solar effect on the starshade brightness to be edge glint.

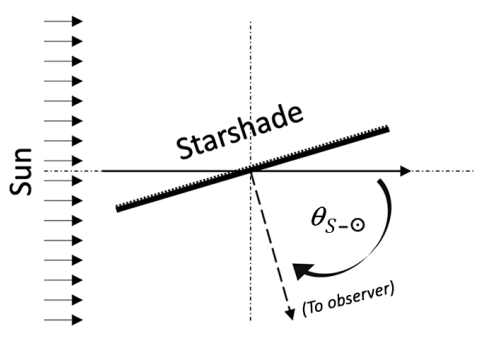

Fig. 1 Schematic of starshade plane-Sun angle $\theta_{S-\odot}$, where the Sun illumination direction is marked by arrows and the starshade plane normal is dashed. An angle of 90 deg corresponds to the Sun being perpendicular to the starshade and 180 deg to the Sun being behind the observer and fully illuminating the starshade surface. 


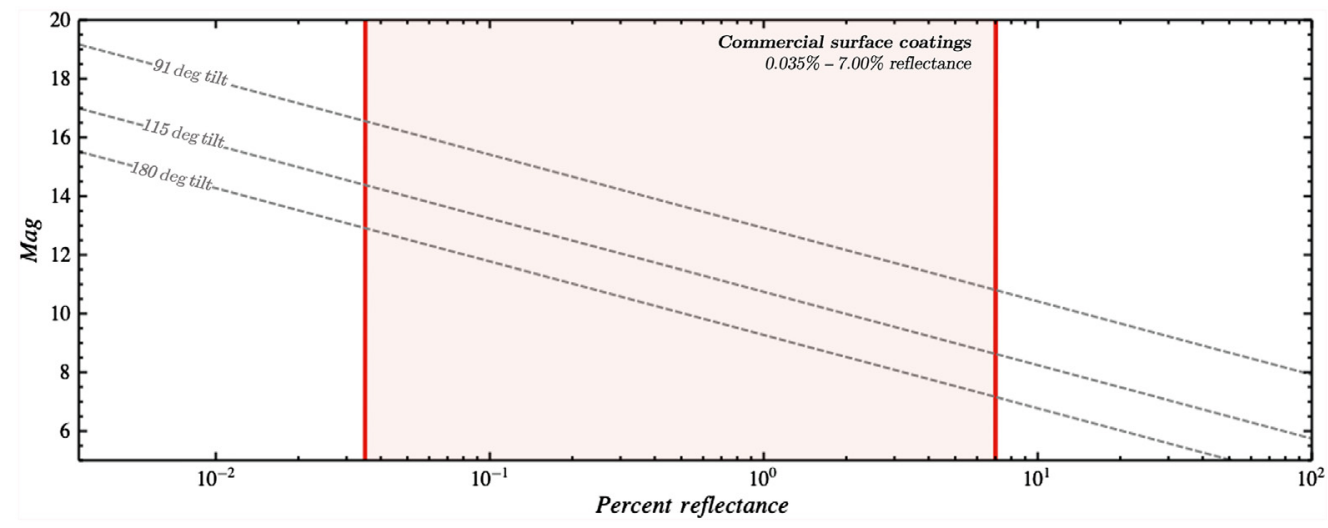

Fig. 2 The starshade surface's apparent magnitude plotted as a function of its surface material reflectance. Each tilt angle corresponds to the starshade plane-Sun angle.

\subsection{Starshade Plane-Target Star Angle}

The ability to deflect the starshade surface normal away from the telescope-target line of sight (LOS) could enable a wider coverage of targets across the sky by limiting Sun-surface illumination and loosening attitude tolerances, therefore, relaxing the Remote Occulter's operational regime. However, as with other starshade missions, preliminary optical error budget analyses indicate that tilting the starshade plane reduces the contrast performance, negatively impacting the ability to obtain meaningful imaging and spectral information from a target.

Off-LOS tilting significantly complicates predicting starshade performance, as light diffracts around petals which no longer hold symmetry or ideal apodization profiles. We evaluate the effect of starshade deflection angles $\theta_{S-T}$, as seen in Fig. 3 on optical performance with the Starshade Imagining Simulation Toolkit for Exoplanet Reconnaissance (SISTER). ${ }^{26}$ Following after our previously developed approach for simulating the optical performance of a tilted starshade, we conduct high-fidelity imaging simulations (30-min exposure with 39-m aperture) of temperate planets around a Sun-like star $(\mathrm{G} 2 \mathrm{~V})$ at $10 \mathrm{pc}$ and record the resultant signal-to-noiseratio (SNR) of an exo-Earth placed at 1 AU radius. As seen in Fig. 4, the starshade can be safely tilted up to $30 \mathrm{deg}$, after which a sigmoidal drop in performance is observed. Although acceptable SNRs could be extracted from larger tilts, we impose a strict requirement to accommodate for unknown loss effects, challenging targets, and spectral variation of target stars in a mission environment.

\subsection{Local Zenith-Sun Angle}

Observation shows that the night sky at high mountain observatories is fully dark at zenith when the Sun is at least $18 \mathrm{deg}$ below the horizon, or equivalently $108 \mathrm{deg}$ off-zenith, here defined as $\theta_{z-\odot}$ as shown in Fig. 5. The sky brightness has been shown to fall by three magnitudes as the $\theta_{Z-\odot}$ increases from $102 \mathrm{deg}$ to $108 \mathrm{deg} .{ }^{27}$ Though observations are possible before the sky

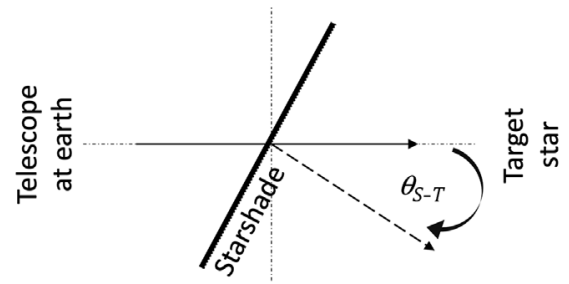

Fig. 3 Schematic of the starshade plane-target angle $\theta_{S-T}$, where the line of sight is marked by the line connecting the telescope at Earth and the target star. The target-facing starshade plane is marked with a dashed arrow and a dotted surface. 

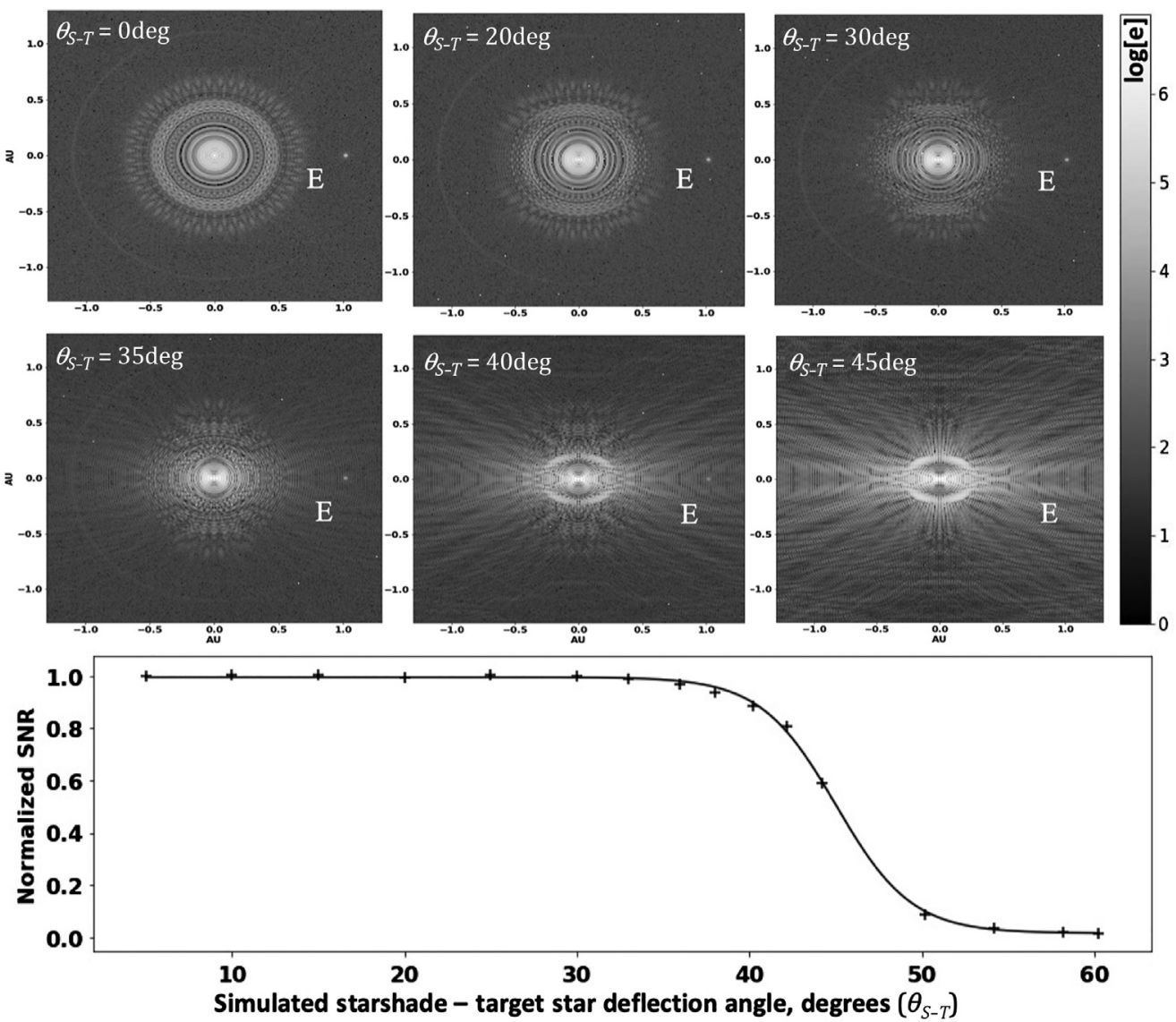

Fig. 4 Remote Occulter sensitivity to $\theta_{S-T}$ deflection angles. (a) SISTER results for a 30-min exposure of a G2V solar system at a distance of $10 \mathrm{pc}$ with 0 exozodi at various starshade deflection angles $\theta_{S-T}$. An exo-Earth is placed at 3 o'clock spaced to $1 \mathrm{AU}$. The $X$ and $Y$ axes define the window of the simulation in $\mathrm{AU}$, and the colorbar corresponds to the total electron counts in log scale. (b) Simulated image SNRs normalized to 0 deg tilt plotted as a function of deflection angle.

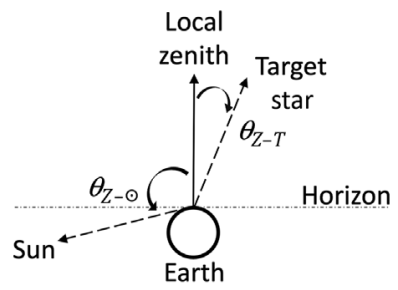

Fig. 5 A schematic of local zenith-Sun angle $\theta_{Z-\odot}$ and local zenith-target star angle $\theta_{Z-T}$ as measured from the observatory's local zenith. We set $\theta_{z-\odot}$ to be below the horizon (dashed) by at least $18 \mathrm{deg}$.

is fully dark and scattered sunlight is not the dominant sky brightness at all wavelengths, we constrain the observing window by assuming this is a strict requirement.

\subsection{Local Zenith-Target Star Angle}

The ELT, TMT, and GMT permit observations from near- or at-zenith down to $20 \mathrm{deg},{ }^{28} 25 \mathrm{deg},{ }^{16}$ and $30 \mathrm{deg}^{17}$ above the horizon, corresponding to a $\theta_{Z-T}$ angle of $70 \mathrm{deg}, 65 \mathrm{deg}$, and $60 \mathrm{deg}$, respectively. We therefore set $\theta_{Z-T_{\max }}<60 \mathrm{deg}$. Additional considerations relating to specific telescope configurations like zenith constraints produced by Alt-azimuth mounts and instruments are provided in Sec. 4. 


\subsection{Intuitive Geometric Model}

The result of these combined geometric constraints is difficult to visualize in three dimensions. We present a model of the observable regions in a stretch of sky sliced across the ecliptic plane, driving an intuitive understanding of each geometric constraint's, detailed in Table 1, effect on the observable sky window in Fig. 6. The constraint of the telescope-zenith angle projects an observable cone onto the celestial sphere that sweeps across the sky between Sun-down and Sunup, while $\theta_{z-\odot}>108 \mathrm{deg}$. The available observation time is then the duration a given star's position satisfies $\theta_{Z-T}$, bounded by $\theta_{z-\odot}$ 's definition of night. On the other hand, the unobservable bounds produced by the starshade plane-Sun and starshade plane-target star deflection constraints are effectively constant over a sidereal day, being entirely dependent on the relative

Table 1 A summary of all geometric requirements. Each row provides a description for an angle and the maximum/ minimum value.

\begin{tabular}{llc}
\hline \hline Angle & \multicolumn{1}{c}{ Description } & Value (deg) \\
\hline$\theta_{Z-T}$ & Local zenith-target star & $<60$ \\
$\theta_{S-\odot}$ & Starshade plane-Sun & $<89$ \\
$\theta_{S-T}$ & Starshade plane-target star & $<30$ to 40 \\
$\theta_{Z-\odot}$ & Local zenith-Sun & $>108$ \\
\hline \hline
\end{tabular}

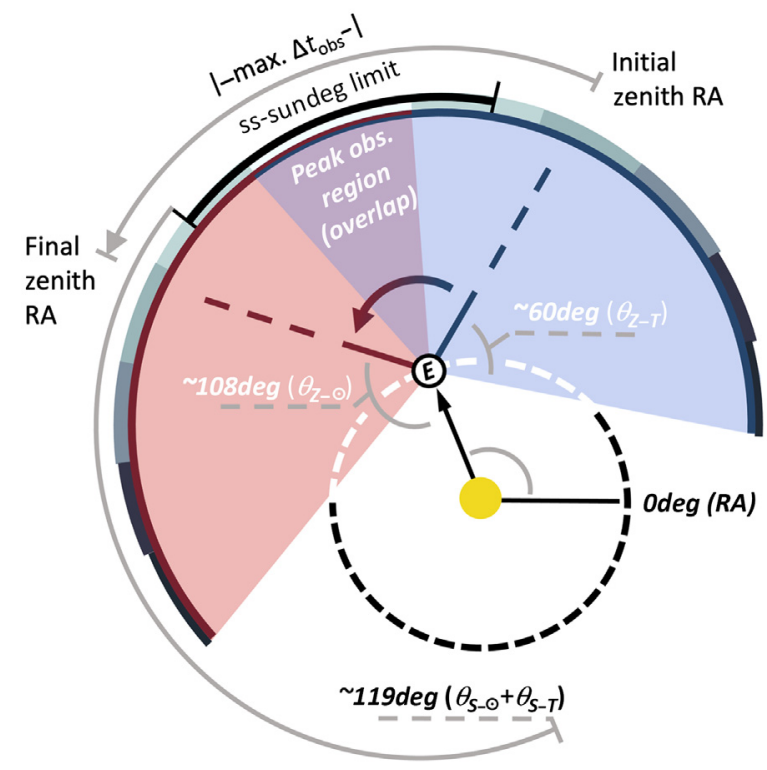

Fig. 6 Geometric schematic of the observable window sliced across the ecliptic plane. Each night's observable sky region is centered on the Sun-Earth RA, where the Sun is marked in yellow and the Earth with an "E." As the Earth rotates over a given night (as defined by the Sun being $>18$ deg below the horizon), the conical regions of observable sky whose zenith-target angle $\theta_{Z-T}$ is $<60 \mathrm{deg}$ and satisfy limiting starshade constraints $\theta_{S-\odot}$ and $\theta_{S-T}$ may be observed. These regions are marked for the start and end of night as blue and red arc-sectors. For a given target coordinate, the starshade surface is best deflected to ensure $\theta_{S-\odot}<89$ deg by a rotation in the plane formed by starshade-target and starshade-Sun vectors up to $\theta_{S-T_{\max }}=30 \mathrm{deg}$, when the angle between target star and the Sun is $119 \mathrm{deg}$, equal to $\theta_{S-T_{\max }}+\theta_{S-\odot_{\max }}$. These bounds are marked as "ss-Sun limit." The external arc-gradient indicates the observable duration of each potential target position, which linearly increases in direct proportion to RA up to a potential "max. $\Delta t_{\text {obs }}$ " in the purple zenith cone overlap region. Targets cannot be observed in the starshade-Sun constrained zone marked by a black arc. 
Sun-Earth positioning. They produce an unobservable region centered on the hour angle of sidereal midnight, marked in black, when the sun directly faces the starshade.

\section{Observable Sky}

To map the regions and duration over which all geometric requirements are met, we adopt a standard epoch (J2000), generate a right ascension (RA) and declination (DEC) grid to project results onto, and choose an observatory location, here Mauna Kea and Cerro Armazones, the locations of TMT and ELT, respectively, for the proposed Remote Occulter science operation period, starting January 2035. Through reference frame and coordinate conversions, we sum the total accumulated duration that these requirements can be kept for each celestial position on a given night and map the resulting observation window contour.

These numerical checks are iteratively applied in discrete time steps using Python. As per Fig. 7, the Sun's ecliptic and topocentric coordinates are calculated for each day of the year, and the local zenith's projected angle is calculated for each minute in a given sidereal day. As the program steps through the time of night that the solar-zenith constraint is satisfied, a minute of observation is added to each celestial coordinate that satisfies the remaining three geometric constraints. We translate the celestial target positions (RA, $\alpha$ and DEC, $\delta$ ) into geometric angles relative to the time-dependent Sun location $\left(\alpha_{\odot}, \delta_{\odot}\right)$ and projected local zenith $\left(\alpha_{z}, \delta_{z}\right)$, using the following equations based on polar reference frame conversions:

$$
\begin{gathered}
\cos \left(\theta_{Z-\odot}\right)=\sin \left(\lambda_{T}\right) \sin \left(\delta_{s}\right)+\cos \left(\lambda_{T}\right) \cos \left(\delta_{s}\right) \cos \left(\alpha_{s}\right) \\
\cos \left(\theta_{Z-T}\right)=\cos \left(\frac{\pi}{2}-\delta_{Z}\right) \cos \left(\frac{\pi}{2}-\delta\right)+\sin \left(\frac{\pi}{2}-\delta_{Z}\right) \sin \left(\frac{\pi}{2}-\delta\right) \cos \left(\alpha-\alpha_{Z}\right), \\
\cos \left(\theta_{S-\odot}+\theta_{S-T}\right)=\cos \left(\frac{\pi}{2}-\delta_{s}\right) \cos \left(\frac{\pi}{2}-\delta\right)+\sin \left(\frac{\pi}{2}-\delta_{s}\right) \sin \left(\frac{\pi}{2}-\delta\right) \cos \left(\alpha-\alpha_{s}\right) .
\end{gathered}
$$

Figure 8 shows the observable regions in the night sky for March 14, 2035, for observations from Cerro Armazones. As indicated by the red border, the maximum declinatory angles that can be observed are $\sim-90 \mathrm{deg}$ and $30 \mathrm{deg}$, constrained by the limiting boundary produced by $\theta_{Z-T}=60 \mathrm{deg}$, the maximum local zenith-target angle. Limits on RAs are set by the first and last RA that fulfill all four requirements and are primarily constrained by the length of the observable night, produced by $\theta_{Z-\odot}>108 \mathrm{deg}$, and the inner bound of maximal target-Sun angle, $\theta_{S-T}+\theta_{S-\odot}=30 \mathrm{deg}+89 \mathrm{deg}$. The outermost red border reflects at least one of the four geometric constraints is at its limit, whereas different color curves indicate the regions where such conditions can be kept for different time periods. The blue line, for example, contains where observations can be maintained for at least $2 \mathrm{~h}$ over a night.

We then continue to dynamically map the observable night sky for the months of January, April, July, and October for both Mauna Kea and Cerro Armazones. As can be seen in Fig. 9, the observable region sweeps across the sky, covering more than 65\% of the sky for each telescope and over $90 \%$ of the celestial sphere over a year for both the north and south hemisphere telescopes.

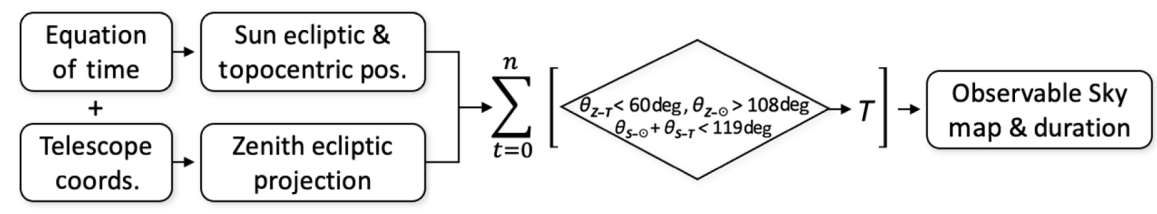

Fig. 7 Flowchart and calculation procedure for our numerical observable map generation method, which produces an observability contour for a given telescope latitude $\left(\lambda_{T}\right)$. Sun-zenith, telescopezenith, combined target-Sun, and checked for suitability at each time-step (every minute of the day $0 \rightarrow n, n=1440$ for each given day of the year). A minute of observation time is added if all geometric requirement statements resolve to "true" at a given coordinate. 


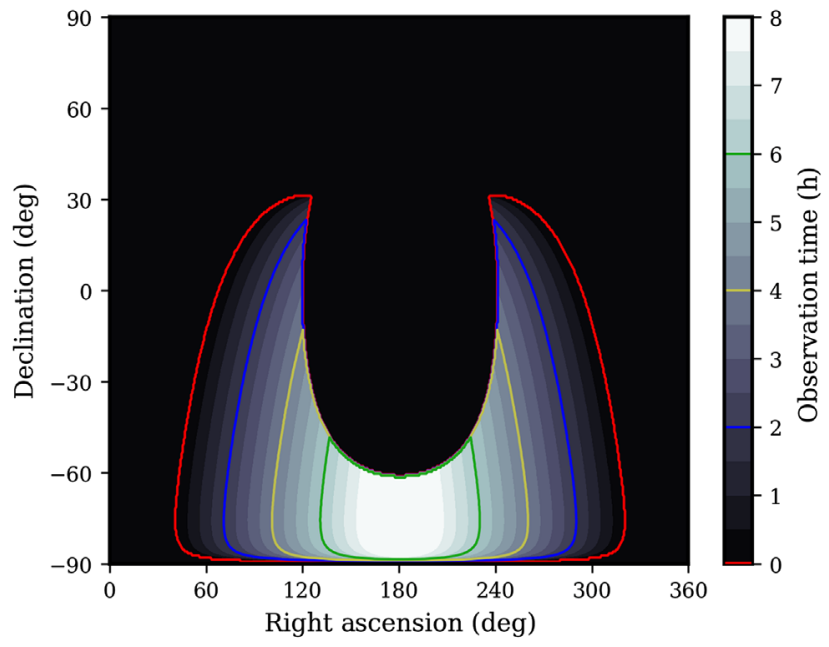

Fig. 8 Observable regions in the night sky for March 14, 2035, for observations from Cerro Armazones. The horizontal axis is 0 deg to $360 \mathrm{deg}$ RA and the vertical axis is -90 deg to +90 deg DEC. The colors correspond to the observation time in hours.
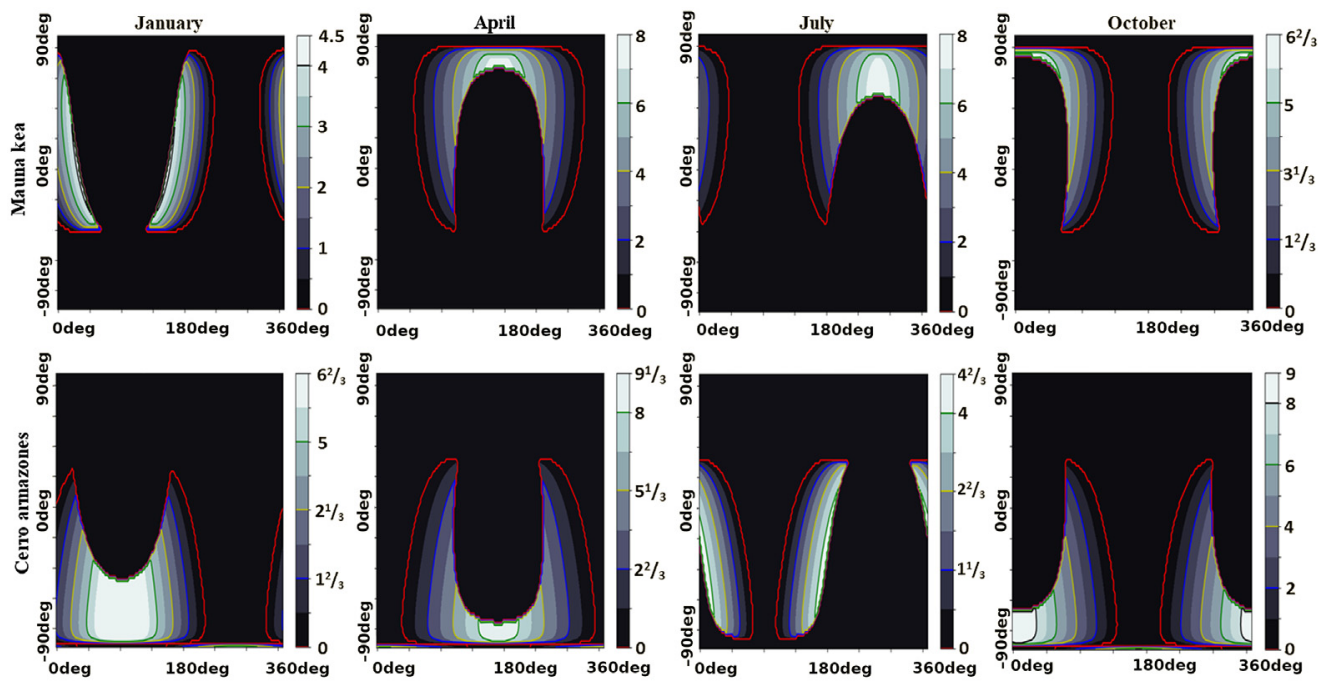

Fig. 9 Observable night sky, showing minutes available for observation each night as indicated by the color bar. The range of each image is 0 deg to 360 deg RA and -90 deg to +90 deg for DEC. Upper set is for Mauna Kea, lower set for Cerro Armazones. Dates are the first of each month, 2035. Each sky-map is colored according its total observation time in hours. Video 1, MOV, $4 \mathrm{MB}$ [URL: https://doi.org/10.1117/1.JATIS.7.2.021212.1] shows a time lapse of the observable sky as seen from Cerro Armazones.

\section{Discussion}

The length of the night directly drives the maximum available observation time and angular width of the overall window. The longest and shortest times to observe occur at the summer and winter solstices depending on the telescope hemisphere. To evaluate the sensitivity of our observable map to the geometric and optical mission constraints, we show in Fig. 10 the result of varied starshade plane-target star angle and local zenith-Sun angle requirements. The maximum combined starshade plane-Sun angle $\left(\theta_{S-\odot}\right)$ and starshade plane-target star angle $\left(\theta_{S-T}\right)$ is seen to inversely correlate with the central unobservable area's size, and the available local zenith-Sun angle range $\left(\theta_{Z-\odot}\right)$ with the observable window width and maximum observation time. As presented in Sec. 2.3, the starshade plane-target star angle could be increased up to 40 deg while delivering a normalized SNR above 0.8. Reduced starshade plane-target star and starshade 

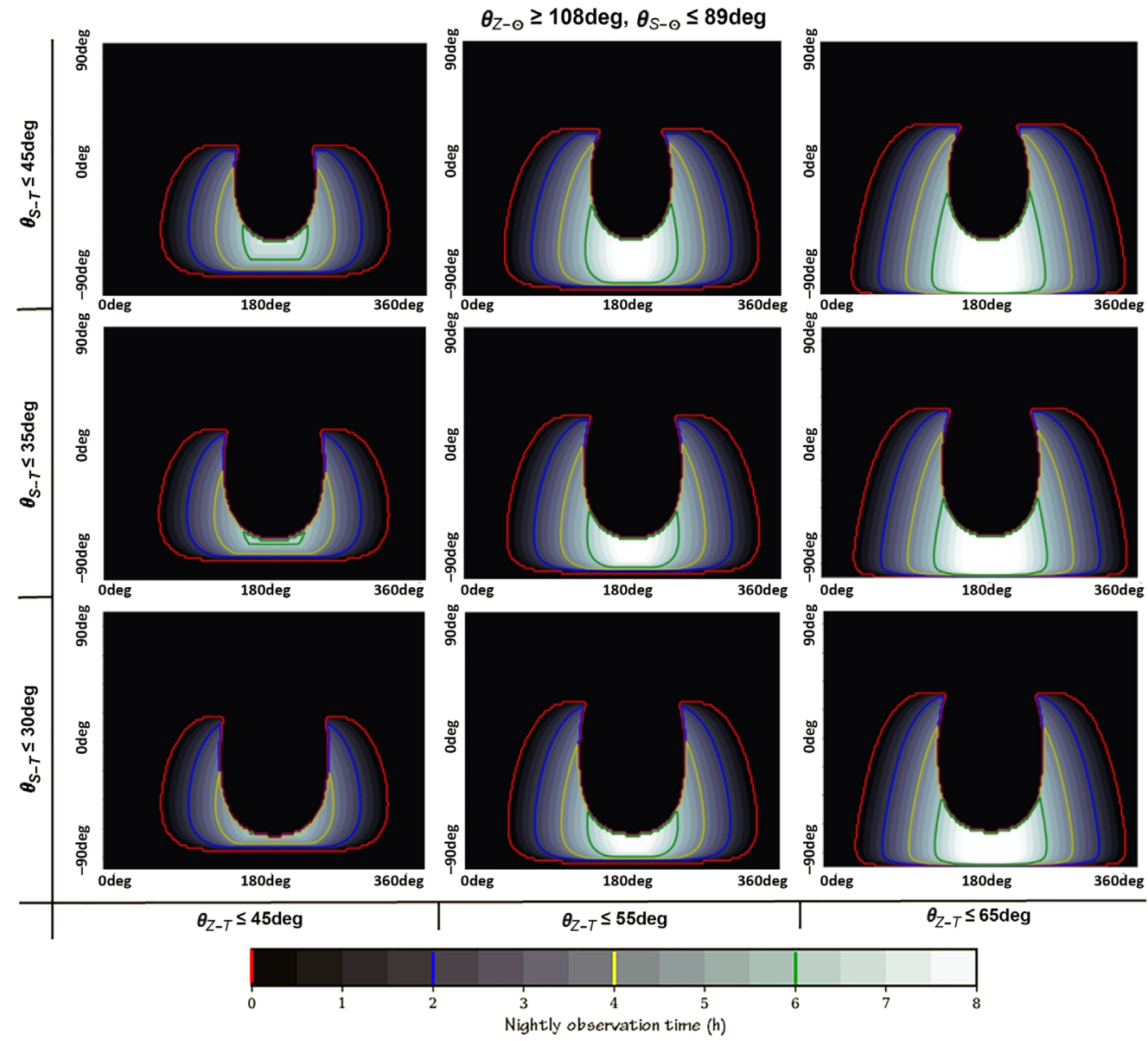

Fig. 10 Sky coverage changes (observable sky) due to the max starshade-target deflection angle, $\theta_{S-T}$, and $\theta_{Z-T}$, the maximum target zenith angle, for observation from Cerro Armazones in April 2035 , the range of each image is 0 deg to 360 deg RA and -90 deg to +90 deg for DEC.

zenith-target star angles limit the observable window's upper and lower declinatory range depending on the time of year. The observable window's progression over a year is available as Video 1.

\section{Summary and Future Work}

In this paper, the observational constraints driven by potentially detrimental optical effects to direct exoplanet imaging with the Remote Occulter mission are reviewed, from which geometric operational requirements have been derived. We develop a numerical method for calculating the observable sky bounds and duration for an Earth-orbiting starshade-coupled ground telescope as constrained by these geometric constraints and present the effects of each geometric constraint on observability and mission architecture and planning. We find that about two-thirds of celestial DECs may be observed with the Remote Occulter at some point in the year for up to $9 \mathrm{~h}$, and that a partnership between both north and southern hemisphere telescopes can enable full sky coverage.

Although the observable window sweeps across all RAs over a year, its time-dependent position and form must be carefully factored into future mission planning studies. Future work will include an array of follow-up studies, from orbital configurations to support minimized stationkeeping costs for observations, retargeting strategies that minimize orbit transfer costs while maintaining observational spatial and time windows, to scheduling optimization for the mission which will inform design activities of mechanical configurations, and operational strategies ultimately leading to a full mission design reference. This could be further expanded by adding or modifying additional constraints; a variety of missions, including the orbiting configurable 
artificial star mission, ${ }^{29}$ could benefit from the mapping of observational regions in their mission planning activities.

\section{Appendices}

\subsection{Appendix A: SISTER}

To create high-fidelity simulations of directly imaged exoplanetary systems, we use the SISTER. SISTER performs these simulations by considering the optical and telescope configurations, and the design of the starshade such as the size and distance from the telescope. A large collection of exoplanetary system parameters such as the spectral type of the host star, the number and type of planets within the system, exozodiacal dust disks, and any background sources such as galaxies or stars. SISTER derives the optical response of the starshade and telecope configuration by performing boundary diffraction modeling to calculate the PSF spatial response. Experimental results show that the resulting PSF's agree to a level of contrast of $10^{-10}$, and it agrees with other simulation tools to a level of $1 \%$. See the SISTER Handbook for further technical details on SISTER.

SISTER allows us to create the PSF response for the Remote Occulter mission. The telescope used is the ELT with an aperture of $39 \mathrm{~m}$. The starshade has 48 petals with the length of each petal set to $24.5 \mathrm{~m}$, and the total diameter is $99 \mathrm{~m}$. The distance between the starshade and the telescope was set to $170,000 \mathrm{~km}$. The PSF response received additional effects by the presence of emission lines in the upper atmosphere, moonlight, scattered starlight, and Earth shine.

To determine how a starshade with a deflection angle effects image quality, we create an astrophysical scene that will remain constant. We select the Sun to be the host star, and it is assigned a range of $10 \mathrm{pc}$ which yields an apparent $\mathrm{V}$ magnitude of 4.83 . We place Earth in an orbit of $1 \mathrm{AU}$ (100 mas planet-star separation at $10 \mathrm{pc}$ ). Excluding the flux from star leakage, we do not turn on any other sources of background such as background galaxies or exozodiacal dust. The only parameter that remains variable is the starshade plane-target star angle $\theta_{S-T}$. Within SISTER, the design of the starshade is stored as $X, Y, Z$ arrays, this allows the user to alter the design of the starshade such as perturbations. To apply a $\theta_{S-T}>0 \mathrm{deg}$, we perform a simple rotation about the $X$ axis of the starshade. Once a new starshade design is built, SISTER creates a new optical response from the new starshade.

As the starshade receives new deflection angles, SISTER creates a new optical response to perform the simulation. Once a new response is created, the $\mathrm{SNR}^{21}$ of Earth is found by utilizing the simulation output data. Once several SNR values were extracted for 16 angles, a sigmoid function was used to fit the data.

\subsection{Appendix B: Starshade Reflection}

We estimate the illuminated starshade magnitude for various surface-Earth phase angles and reflectances by approximating the reflected flux at the starshade surface- to which Lambertian cosine losses may be applied for oblique tilting - and subsequently derive the magnitude at Earth $M$ :

$$
\begin{gathered}
I_{\odot-S}=I_{\odot} \frac{r_{\odot}^{2}}{r_{\odot-S}^{2}}, \\
I_{S-\oplus}=\rho I_{S-\oplus} \frac{r_{S}^{2}}{r_{S-\oplus}^{2}}, \\
M_{S}=-2.5 \log _{10}\left(\frac{I_{S}}{I_{\odot}}\right)+M_{\odot},
\end{gathered}
$$

where $r_{\odot}$ is the radius of the sun (where the value of $I_{\odot}$ is extracted at), $r_{\odot-S}$ is the range from sun to Earth, $r_{S-\oplus}$ is the range from the starshade surface to Earth, $I_{\odot-S}$ is the approximate solar 
irradiance over the starshade surface, $\rho$ is the starshade surface reflectance, and $I_{S-\oplus}$ is the starshade irradiance at Earth.

\section{Acknowledgments}

We appreciate numerous discussions with the Goddard Space Flight Center Orbit Analysis Group, including David Folta, Rizwan Qureshi, Robert Pritchett, Donald Dichmann, Sun Hur-Diaz, and Steven Hughes. We thank the Team-X Laboratory at Jet Propulsion Laboratory for engineering analysis and cost estimation, and the Exoplanet Exploration Program (ExEP) Team for support and technical discussion, especially Gary Blackwood, Keith Warfield, Karl Stapelfeldt, and Erick Mamajek. This work was supported by Goddard Space Flight Center's Internal Research and Development Funds (IRAD) at Goddard and NASA's ExEP supported the Team-X study at JPL and scientific and engineering workshops at Goddard.

\section{References}

1. E. National Academies of Science and Medicine, Exoplanet Science Strategy, The National Academies Press, Washington DC (2018).

2. N. R. Council, New Worlds, New Horizons in Astronomy and Astrophysics, The National Academies Press, Washington DC (2010).

3. Y. Fujii et al., "Exoplanet biosignatures: observational prospects," Astrobiology 18, 739-778 (2018).

4. A. Roberge et al., "The exozodiacal dust problem for direct observations of exo-Earths," Publ. Astron. Soc. Pac. 124, 799-808 (2012).

5. "NASA Exoplanet Archive, Confirmed Planets Table."

6. A. J. Bohn et al., "Two directly imaged, wide-orbit giant planets around the young, solar analog TYC 8998-760-1," Astrophys. J. 898, L16 (2020).

7. M. Kuzuhara et al., "Direct imaging of a cold Jovian exoplanet in orbit around the Sun-like star GJ 504," Astrophys. J. 774, 11 (2013).

8. A. Müller et al., "Orbital and atmospheric characterization of the planet within the gap of the PDS 70 transition disk," Astron. Astrophys. 617, L2 (2018).

9. B. S. Gaudi et al., "The habitable exoplanet observatory (HabEx) mission concept study final report" (2020).

10. T. L. Team, "The LUVOIR mission concept study final report," arXiv:1912.06219 (2019).

11. D. Mawet et al., "Review of small-angle coronagraphic techniques in the wake of groundbased second-generation adaptive optics systems," Proc. SPIE 8442, 844204 (2012).

12. J. R. Males and O. Guyon, "Ground-based adaptive optics coronagraphic performance under closed-loop predictive control," J. Astron. Telesc. Instrum. Syst. 4(1), 019001 (2018).

13. W. Cash, "Analytic modeling of starshades," Astrophys. J. 738, 76 (2011).

14. R. J. Vanderbei, E. Cady, and N. J. Kasdin, "Optimal Occulter design for finding extrasolar planets," Astrophys. J. 665, 794-798 (2007).

15. M. T. Stahl, S. B. Shaklan, and H. P. Stahl, "Preliminary analysis of effect of random segment errors on coronagraph performance," Proc. SPIE 9605, 96050P (2015).

16. G. H. Sanders, "The Thirty Meter Telescope (TMT): an international observatory," J. Astrophys. Astron. 34, 81-86 (2013).

17. J. Fanson et al., "Overview and status of the Giant Magellan Telescope project," Proc. SPIE 10700, 319-332 (2018).

18. ESO, "The E-ELT construction proposal," https://www.eso.org/sci/facilities/eelt/docs/eelt_constrproposal.pdf (2011).

19. K. R. Stapelfeldt, "Extrasolar planets and star formation: science opportunities for future ELTs," in Scientific Requirements Extremely Large Telesc., IAU Symp. 232,P. Whitelock, M. Dennefeld, and B. Leibundgut, Eds., pp. 149-158 (2006).

20. O. Guyon, "Limits of adaptive optics for high-contrast imaging," Astrophys. J. 629, 592-614 (2005). 
21. E. Peretz et al., "Exoplanet imaging performance envelopes for starshade based missions (under review)," J. Astron. Telesc. Instrum. Syst. 7 (2021).

22. J. C. Mather et al., "Orbiting starshade to observe exoplanets with ground-based telescopes (under review)," Nat. Astron (2021).

23. A. Harness et al., "Demonstration of 1e-10 contrast at the inner working angle of a starshade in broadband light and at a flight-like Fresnel number," Proc. SPIE 11117,185-192 (2019).

24. E. Peretz et al., "Exoplanet imaging scheduling optimization for an orbiting 1starshade working with extremely large telescopes," J. Astron. Telesc. Instrum. Syst. 7(1) (2021).

25. A. W. Koenig et al., "Optimal spacecraft orbit design for inertial alignment with ground telescope," in IEEE Aerospace Conf. (2021).

26. S. R. Hildebrandt et al., "SISTER: starshade imaging simulation toolkit for exoplanet reconnaissance," J. Astron. Telesc. Instrum. Syst. 7 (2021).

27. F. Patat, O. S. Ugolnikov, and O. V. Postylyakov, "UBVRI twilight sky brightness at ESO-Paranal," Astron. Astrophys. 455, 385-393 (2006).

28. "ESO—E-ELT enclosure," https://www.eso.org/sci/facilities/eelt/telescope/dome/ (2009).

29. E. Peretz et al., "Orbiting configurable artificial star (ORCAS) for visible adaptive optics from the ground," Bull. Am. Astron. Soc. 51, 284 (2019).

Eliad Peretz is a technology program manager and leads the smallsat development lab at Goddard Space Flight Center, He received his $\mathrm{PhD}$ in mechanical aerospace engineering with a minor in applied physics from Cornell University, and has been with GSFC since 2015.

John C. Mather is the senior project scientist on the James Webb Space Telescope. He is also a senior astrophysicist in the Observational Cosmology Laboratory located at NASA's Goddard Space Flight Center.

Lucas Pabarcius is a NASA Pathways student trainee in attitude control systems at Goddard Space Flight Center and a junior undergraduate student studying physics and astronomy at Wesleyan University.

Sara Seager is the Class of 1941 Professor of Planetary Science, Professor of Physics, and Professor of Aeronautics and Astronautics at the Massachusetts Institute of Technology. She received her PhD from Harvard University.

Stuart Shaklan is the supervisor of the High Contrast Imaging Group in the Optics Section of the Jet Propulsion Laboratory. He received his PhD in optics at the University of Arizona in 1989 and has been with JPL since 1991.

Sergi Hildebrandt is a scientist at the Jet Propulsion Laboratory and lecturer at the California Institute of Technology. He received his $\mathrm{PhD}$ in theoretical physics at the University of Barcelona. He has worked in the data analysis of the cosmic microwave background from space and ground missions, adaptive optics in the visible, and more recently on leading the development of SISTER, a user friendly, open source, project that generates starshade simulations with very high fidelity.

Phil Willems is an optical engineer at the Jet Propulsion Laboratory, where he is the manager of the S5 Starshade Technology Development Activity. He received his BS degree in physics from the University of Wisconsin-Madison in 1988, and his $\mathrm{PhD}$ in physics from the California Institute of Technology in 1997.

Kevin Hall is currently an astrophysics research assistant within the Department of Astronomy at the University of Maryland. He received his BS degree in astronomy and physics in 2019. 